\title{
The Reaction of Sulfonyl Isocyanates with Vinyl Ethers
}

By Dr. F. Effenberger and Dipl.-Chem. R. Gleiter

Institut für Organische Chemie und Organisch-chemische Technologie derTechnischen Hochschule Stuttgart (Germany)

Sulfonyl isocyanates (1) [1], e.g. $p$-toluenesulfonyl isocyanate, react very rapidly and quantitatively with vinyl ethers giving $\mathrm{N}$-substituted 4-alkoxyacetidin-2-ones (2) or $\mathrm{N}$-substituted ß-alkoxyacrylamides (3).

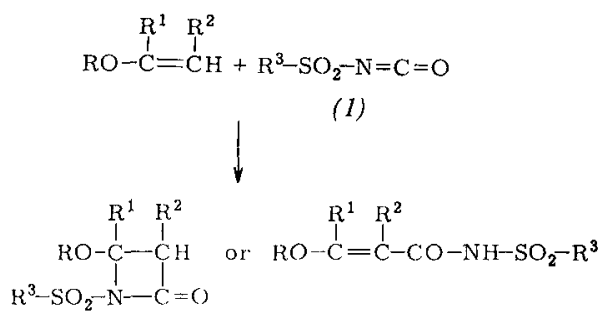

(2)

(3)

\begin{tabular}{|c|c|c|c|c|c|c|}
\hline \multicolumn{4}{|c|}{ Substituents } & $\begin{array}{l}\text { React. } \\
\text { teirip. } \\
{\left[{ }^{\circ} \mathrm{C}\right]}\end{array}$ & Product & $\begin{array}{l}\text { M.p. } \\
{\left[{ }^{\circ} \mathrm{C}\right]}\end{array}$ \\
\hline $\mathrm{C}_{2} \mathrm{H}_{5}$ & $\mathbf{H}$ & $\mathrm{H}$ & $\mathrm{CH}_{3} \cdots \mathrm{C}_{6} \mathrm{H}_{4}$ & 20 & (2) & $56-63$ \\
\hline $\mathrm{C}_{2} \mathrm{H}_{5}$ & $\mathbf{H}$ & $\mathrm{CH}_{3}$ & $\mathrm{CH}_{3}-\mathrm{C}_{6} \mathrm{H}_{4}$ & 20 & $(3)+(2)$ & - \\
\hline $\mathrm{C}_{2} \mathrm{H}_{5}$ & $\mathbf{H}$ & $\mathrm{C}_{2} \mathrm{H}_{5}$ & $\mathrm{CH}_{3}-\mathrm{C}_{6} \mathrm{H}_{4}$ & 20 & (2) & $76-78$ \\
\hline $\mathrm{C}_{2} \mathrm{H}_{5}$ & $\mathbf{H}$ & $\mathrm{C}_{2} \mathrm{H}_{5}$ & $\mathrm{CH}_{3}-\mathrm{C}_{6} \mathrm{H}_{4}$ & 80 & (3) & -- \\
\hline $\mathrm{C}_{4} \mathrm{H}_{9}$ & $\mathbf{H}$ & $\mathrm{H}$ & $\mathrm{CH}_{3}-\mathrm{C}_{6} \mathrm{H}_{4}$ & 20 & $(2)+(3)$ & 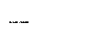 \\
\hline $\mathrm{C}_{2} \mathrm{H}_{5}$ & $\mathbf{H}$ & $\mathrm{C}_{2} \mathrm{H}_{5}$ & $\mathrm{Cl}$ & $15-17$ & (3) & -- \\
\hline \multicolumn{3}{|c|}{ Dihydropyran } & $\mathrm{CH}_{3} \cdot \mathrm{C}_{6} \mathrm{H}_{4}$ & 20 & $(2)+(3)$ & $65-70$ \\
\hline \multicolumn{3}{|c|}{ Dihydropyran } & $\mathrm{CH}_{3}-\mathrm{C}_{6} \mathrm{H}_{4}$ & 80 & (3) & $204--205$ \\
\hline \multicolumn{3}{|c|}{ Dihydropyran } & $\mathrm{Cl}$ & $15 \quad 20$ & (3) & $88--90$ \\
\hline
\end{tabular}

Formation of (2) or (3) depends on the substituents as well as on the reaction conditions. Viscous oils are often obtained when mixtures of (2) and (3) are formed, but (2) or (3) alone is almost always a crystalline compound. The characteristic carbonyl frequency for (2) lies between 1780 and $1800 \mathrm{~cm}^{-1}$, for (3) between 1670 and $1730 \mathrm{~cm}^{-1}$.
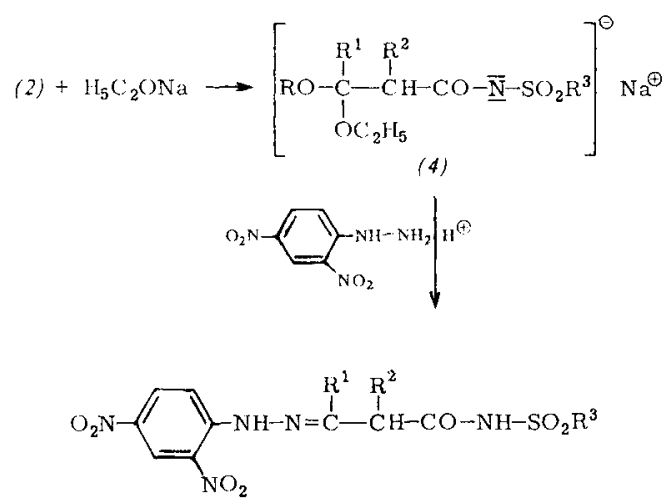

With sodium ethoxide, (2) gives salts of $N$-substituted $\beta, \beta$ dialkoxypropionamides (4), which form hydrazones with 2,4-dinitrophenylhydrazine.

Received, March 26th, 1963 [Z 479/309 IE]

[1] H. Krzikalla, German Pat. 817602 (Oct. 18th, 1951); R. Graf, Chem. Ber. 89, 1071 (1956) 